\title{
Implications of a deeper level explanation of the deBroglie-Bohm version of quantum mechanics
}

\author{
G. Grössing • S. Fussy • J. Mesa Pascasio • \\ H. Schwabl
}

Received: 2 January 2015 / Accepted: 1 February 2015 / Published online: 14 February 2015

(C) Chapman University 2015

\begin{abstract}
Elements of a "deeper level" explanation of the deBroglie-Bohm (dBB) version of quantum mechanics are presented. Our explanation is based on an analogy of quantum wave-particle duality with bouncing droplets in an oscillating medium, the latter being identified as the vacuum's zero-point field. A hydrodynamic analogy of a similar type has recently come under criticism by Richardson et al. (On the analogy of quantum wave-partile duality with bouncing droplets, 2014), because despite striking similarities at a phenomenological level the governing equations related to the force on the particle are evidently different for the hydrodynamic and the quantum descriptions, respectively. However, said differences are not relevant if a radically different use of said analogy is being made, thereby essentially referring to emergent processes in our model. If the latter are taken into account, one can show that the forces on the particles are identical in both the $\mathrm{dBB}$ and our model. In particular, this identity results from an exact matching of our emergent velocity field with the Bohmian "guiding equation". One thus arrives at an explanation involving a deeper, i.e., subquantum, level of the dBB version of quantum mechanics. We show in particular how the classically local approach of the usual hydrodynamical modeling can be overcome and how, as a consequence, the configuration-space version of dBB theory for $N$ particles can be completely substituted by a "superclassical" emergent dynamics of $N$ particles in real three-dimensional space.
\end{abstract}

Keywords Quantum mechanics · Hydrodynamics · DeBroglie-Bohm theory · Guiding equation · Configuration space $\cdot$ Zero-point field

\section{Mathematics Subject Classification $\quad 82 \mathrm{C} 10$}

\section{Introduction}

The Schrödinger equation for $N>1$ particles does not describe a wave function in ordinary three-dimensional space, but instead in an abstract $3 \mathrm{~N}$-dimensional space. For quantum realists, including Schrödinger and Einstein, for example, this has always been considered as "indigestible". This holds even more so for a realist, causal approach

G. Grössing · S. Fussy · J. Mesa Pascasio $(\varangle) \cdot$ H. Schwabl

Austrian Institute for Nonlinear Studies, Akademiehof, Friedrichstr. 10, 1010 Vienna, Austria

e-mail: johannes.mesa@gmx.at

G. Grössing

e-mail: ains@chello.at 
to quantum phenomena such as the deBroglie-Bohm $(\mathrm{dBB})$ version of quantum mechanics. David Bohm himself has admitted this, calling it a "serious problem": "While our theory can be extended formally in a logically consistent way by introducing the concept of a wave in a $3 N$-dimensional space, it is evident that this procedure is not really acceptable in a physical theory, and should at least be regarded as an artifice that one uses provisionally until one obtains a better theory in which everything is expressed once more in ordinary three-dimensional space" [1]. (For more detailed accounts of this discussion already in the early years of quantum mechanics, see $[17,18]$.)

In the present paper, we shall refer to our attempt towards such a "better theory" in terms of a deeper level, i.e., subquantum, approach to the $\mathrm{dBB}$ theory, and thus to quantum theory in general. In fact, with our model, we have in a series of papers already obtained several essential elements of nonrelativistic quantum theory $[8,9,13,14]$. They derive from the assumption that a particle of energy $E=\hbar \omega$ is actually an oscillator of angular frequency $\omega$ phase-locked with the zero-point oscillations of the surrounding environment, the latter containing both regular and fluctuating components and being constrained by the boundary conditions of the experimental setup via the buildup and maintenance of standing waves. The particle in this approach is an off-equilibrium steady-state oscillation maintained by a constant throughput of energy provided by the ("classical") zero-point energy field. We have, for example, applied the model to the case of interference at a double slit, thereby obtaining the exact quantum mechanical probability density distributions on a screen behind the double slit, the average trajectories (which because of the averaging are shown to be identical to the Bohmian ones), and the involved probability density currents. Our whole model is constructed in close analogy to the bouncing/walking droplets above the surface of a vibrated liquid in the experiments first performed by Couder and Fort [4,5], Fort and co-workers [6], which in many respects can serve as a classical prototype guiding our intuition for the modeling of quantum systems.

However, there are also obvious differences between the mentioned physics of classical bouncers/walkers on the one hand, and the hydrodynamic-like models for quantum systems like our own model or the dBB on the other hand. In a recent paper, Richardson et al. [20] have probed more thoroughly into the hydrodynamic analogy of dBB-type quantum wave-particle duality with that of the classical bouncing droplets. Apart from the obvious difference in that Bohmian theory is distinctly nonlocal, whereas droplet-surface interactions are rooted in classical hydrodynamics and thus in a manifestly local theory, Richardson et al. focus on the following observation: the evidently different nature of the Bohmian force upon a quantum particle as compared to the force that a surface wave exerts upon a droplet. In fact, wherever the probability density in the $\mathrm{dBB}$ picture is close to zero, the quantum force becomes singular and will very quickly push any particle away from that area. Conversely, the hydrodynamic force directs the droplet into the trough of the wave! So, the probability of finding a droplet in the minima never reaches zero as it does for a quantum particle. The authors conclude that these discrepancies between the two models highlight "a major difference between the hydrodynamic force and the quantum force" [20].

Although these authors generally recover in numerical hydrodynamic simulations the results of the Paris group (later confirmed also by the group of Bush [3] at MIT) on single-slit diffraction and double-slit interference, they also point out the (already known) striking contrast between the trajectory behaviors for the bouncing droplet systems and dBB-type quantum mechanics, respectively. Whereas the latter exhibits the well-known no-crossing property, the trajectories of the former do to a large extent cross each other. So, again, the physics in the two models is apparently fundamentally different, despite some striking similarities on a phenomenological level. As to the differences, one may very well expect that they will even become more severe when moving from one-particle to $N$-particle systems.

So, all in all, the paper by Richardson et al. [20] cautions against the assumption of too close a resemblance of bouncer/walker systems and the hydrodynamic-like modeling of quantum systems like the $\mathrm{dBB}$, with their main argument being that the hydrodynamic force on a droplet strikingly contrasts with the quantum force on a particle in the $\mathrm{dBB}$ theory. However, we shall here argue against the possible conclusion that one has thus reached the limits of applicability of the hydrodynamic bouncer analogy for quantum modeling. On the contrary, as we have already pointed out in previous papers, it is a more detailed model inspired by the bouncer/walker experiments that can show the fertility of said analogy. It enables us to show that our model, being of the type of an "emergent quantum mechanics" [10,11], can provide a deeper level explanation of the dBB version of quantum mechanics (Sect. 2). Moreover, as we shall also show, it turns out to provide an identity of an emergent force on the bouncer in our 
hydrodynamic-like model with the quantum force in Bohmian theory (Sect. 3). Finally, in Sect. 4, we shall discuss the "price" to be paid to arrive at our explanation of dBB theory in that some kind of nonlocality, or a certain "systemic nonlocality", has to be admitted in the model from the start. However, the simplicity and elegance of our derived formalism, combined with arguments about the reasonableness of a corresponding hydrodynamic-like modeling, will show that our approach may be a viable one w.r.t. understanding the emergence of quantum phenomena from the interactions and contextualities provided by the combined levels of classical boundary conditions and those of a subquantum domain.

\section{Identity of the emergent kinematics of $N$ bouncers in real three-dimensional space with the configuration -space version of deBroglie-Bohm theory for $N$ particles}

Consider one particle in an $n$-slit system. In quantum mechanics, as well as in our quantum-like modeling via an emergent quantum mechanics approach, one can write down a formula for the total intensity distribution $P$ which is very similar to the classical formula. For the general case of $n$ slits, it holds with phase differences $\varphi_{i j}=\varphi_{i}-\varphi_{j}$ that

$P=\sum_{i=1}^{n}\left(P_{i}+\sum_{j=i+1}^{n} 2 R_{i} R_{j} \cos \varphi_{i j}\right)$,

where the phase differences are defined over the whole domain of the experimental setup. Apart from the role of the relative phase with important implications for the discussions on nonlocality [14], there is one additional ingredient that distinguishes (1) from its classical counterpart, namely the "dispersion of the wavepacket". As in our model the "particle" is actually a bouncer in a fluctuating wave-like environment, i.e., analogously to the bouncers of Couder and Fort's group, one does have some (e.g., Gaussian) distribution, with its center following the Ehrenfest trajectory in the free case, but one also has a diffusion to the right and to the left of the mean path which is just due to that stochastic bouncing. Thus the total velocity field of our bouncer in its fluctuating environment is given by the sum of the forward velocity $\mathbf{v}$ and the respective diffusive velocities $\mathbf{u}_{\mathrm{L}}$ and $\mathbf{u}_{\mathrm{R}}$ to the left and the right. As for any direction $i$ the diffusion velocity $\mathbf{u}_{i}=D \frac{\nabla_{i} P}{P}$ does not necessarily fall off with the distance, one has long effective tails of the distributions which contribute to the nonlocal nature of the interference phenomena [14]. In sum, one has three, distinct velocity (or current) channels per slit in an $n$-slit system.

We have previously shown $[7,15]$ how one can derive the Bohmian guidance formula from our bouncer/walker model. To recapitulate, we recall the basics of that derivation here. Introducing classical wave amplitudes $R\left(\mathbf{w}_{i}\right)$ and generalized velocity field vectors $\mathbf{w}_{i}$, which stand for either a forward velocity $\mathbf{v}_{i}$ or a diffusive velocity $\mathbf{u}_{i}$ in the direction transversal to $\mathbf{v}_{i}$, we account for the phase-dependent amplitude contributions of the total system's wave field projected on one channel's amplitude $R\left(\mathbf{w}_{i}\right)$ at the point $(\mathbf{x}, t)$ in the following way: We define a conditional probability density $P\left(\mathbf{w}_{i}\right)$ as the local wave intensity $P\left(\mathbf{w}_{i}\right)$ in one channel (i.e., $\left.\mathbf{w}_{i}\right)$ upon the condition that the totality of the superposing waves is given by the "rest" of the $3 n-1$ channels (recalling that there are three velocity channels per slit). The expression for $P\left(\mathbf{w}_{i}\right)$ represents what we have termed "relational causality": any change in the local intensity affects the total field, and vice versa, any change in the total field affects the local one. In an $n$-slit system, we thus obtain for the conditional probability densities and the corresponding currents, respectively, i.e., for each channel component $i$,

$$
\begin{aligned}
P\left(\mathbf{w}_{i}\right) & =R\left(\mathbf{w}_{i}\right) \hat{\mathbf{w}}_{i} \cdot \sum_{j=1}^{3 n} \hat{\mathbf{w}}_{j} R\left(\mathbf{w}_{j}\right) \\
\mathbf{J}\left(\mathbf{w}_{i}\right) & =\mathbf{w}_{i} P\left(\mathbf{w}_{i}\right), \quad i=1, \ldots, 3 n,
\end{aligned}
$$

with

$$
\cos \varphi_{i, j}:=\hat{\mathbf{w}}_{i} \cdot \hat{\mathbf{w}}_{j} .
$$


Consequently, the total intensity and current of our field read as

$$
\begin{aligned}
& P_{\mathrm{tot}}=\sum_{i=1}^{3 n} P\left(\mathbf{w}_{i}\right)=\left(\sum_{i=1}^{3 n} \hat{\mathbf{w}}_{i} R\left(\mathbf{w}_{i}\right)\right)^{2} \\
& \mathbf{J}_{\mathrm{tot}}=\sum_{i=1}^{3 n} \mathbf{J}\left(\mathbf{w}_{i}\right)=\sum_{i=1}^{3 n} \mathbf{w}_{i} P\left(\mathbf{w}_{i}\right),
\end{aligned}
$$

leading to the emergent total velocity

$$
\mathbf{v}_{\mathrm{tot}}=\frac{\mathbf{J}_{\mathrm{tot}}}{P_{\mathrm{tot}}}=\frac{\sum_{i=1}^{3 n} \mathbf{w}_{i} P\left(\mathbf{w}_{i}\right)}{\sum_{i=1}^{3 n} P\left(\mathbf{w}_{i}\right)} .
$$

In $[7,15]$, we have shown with the example of $n=2$, i.e., a double-slit system, that Eq. (7) can equivalently be written in the form

$\mathbf{v}_{\mathrm{tot}}=\frac{R_{1}^{2} \mathbf{v}_{1}+R_{2}^{2} \mathbf{v}_{2}+R_{1} R_{2}\left(\mathbf{v}_{1}+\mathbf{v}_{2}\right) \cos \varphi+R_{1} R_{2}\left(\mathbf{u}_{1}-\mathbf{u}_{2}\right) \sin \varphi}{R_{1}^{2}+R_{2}^{2}+2 R_{1} R_{2} \cos \varphi}$.

The trajectories or streamlines, respectively, are obtained according to $\dot{\mathbf{x}}=\mathbf{v}_{\text {tot }}$ in the usual way by integration. As first shown in [13], by re-inserting the expressions for convective and diffusive velocities, respectively, i.e., $\mathbf{v}_{i}=\frac{\nabla S_{i}}{m}, \mathbf{u}_{i}=-\frac{\hbar}{m} \frac{\nabla R_{i}}{R_{i}}$, one immediately identifies Eq. (8) with the Bohmian guidance formula. Naturally, employing the Madelung transformation for each path $j(j=1$ or 2$)$,

$\psi_{j}=R_{j} \mathrm{e}^{i S_{j} / \hbar}$,

and thus $P_{j}=R_{j}^{2}=\left|\psi_{j}\right|^{2}=\psi_{j}^{*} \psi_{j}$, with $\varphi=\left(S_{1}-S_{2}\right) / \hbar$, and recalling the usual trigonometric identities such as $\cos \varphi=\frac{1}{2}\left(\mathrm{e}^{i \varphi}+\mathrm{e}^{-i \varphi}\right)$, one can rewrite the total average current immediately in the usual quantum mechanical form as

$$
\begin{aligned}
\mathbf{J}_{\mathrm{tot}} & =P_{\mathrm{tot}} \mathbf{v}_{\mathrm{tot}} \\
& =\left(\psi_{1}+\psi_{2}\right)^{*}\left(\psi_{1}+\psi_{2}\right) \frac{1}{2}\left[\frac{1}{m}\left(-i \hbar \frac{\nabla\left(\psi_{1}+\psi_{2}\right)}{\left(\psi_{1}+\psi_{2}\right)}\right)+\frac{1}{m}\left(i \hbar \frac{\nabla\left(\psi_{1}+\psi_{2}\right)^{*}}{\left(\psi_{1}+\psi_{2}\right)^{*}}\right)\right] \\
& =-\frac{i \hbar}{2 m}\left[\Psi^{*} \nabla \Psi-\Psi \nabla \Psi^{*}\right]=\frac{1}{m} \operatorname{Re}\left\{\Psi^{*}(-i \hbar \nabla) \Psi\right\},
\end{aligned}
$$

where $P_{\mathrm{tot}}=\left|\psi_{1}+\psi_{2}\right|^{2}=:|\Psi|^{2}$.

Equation (7) has been derived for one particle in an $n$-slit system. However, it is straightforward to extend this derivation to the many-particle case. Due to the purely additive terms in the expressions for the total current and total probability density, respectively, also for $N$ particles, the only difference now is that the currents' nabla operators have to be applied at all of the locations of the respective $N$ particles, thus providing the quantum mechanical formula

$\mathbf{J}_{\mathrm{tot}}(N)=\sum_{i=1}^{N} \frac{1}{m_{i}} \operatorname{Re}\left\{\Psi(t)^{*}\left(-i \hbar \nabla_{i}\right) \Psi(t)\right\}$, 
where $\Psi(t)$ now is the total $N$-particle wave function, whereas the total velocity fields are given by

$\mathbf{v}_{i}(t)=\frac{\hbar}{m_{i}} \operatorname{Im} \frac{\nabla_{i} \Psi(t)}{\Psi(t)} \forall i=1, \ldots, N$.

Note that this result is similar in spirit to that of Norsen et al. [17,18] who with the introduction of a conditional wave function $\tilde{\psi}_{i}$, as opposed to the configuration-space wave function $\Psi$, rewrite the guidance formula, for each particle, in terms of the $\tilde{\psi}_{i}$ :

$$
\frac{\mathrm{d} X_{i}(t)}{\mathrm{d} t}=\left.\left.\frac{\hbar}{m_{i}} \operatorname{Im} \frac{\nabla \Psi}{\Psi}\right|_{\boldsymbol{x}=\boldsymbol{X}(\mathbf{t})} \equiv \frac{\hbar}{m_{i}} \operatorname{Im} \frac{\nabla \tilde{\psi}_{i}}{\tilde{\psi}_{i}}\right|_{x=X_{i}(t)},
$$

where the $X_{i}$ denote the location of one specific particle and $\mathbf{X}(t)=\left\{X_{1}(t), \ldots, X_{N}(t)\right\}$ the actual configuration point. Thus, in this approach, each $\tilde{\psi}_{i}$ can be regarded as a wave propagating in physical three-dimensional space.

In sum, with our introduction of a conditional probability $P\left(\mathbf{w}_{i}\right)$ for channels $\mathbf{w}_{i}$, which include subquantum velocity fields, we obtain the guidance formula also for $N$-particle systems. Therefore, what looks like the necessity in the $\mathrm{dBB}$ theory to superpose wave functions in configuration space in order to provide an "indigestible" guiding wave, can equally be obtained by superpositions of all relational amplitude configurations of waves in real threedimensional space. The central ingredient for this to be possible is to consider the emergence of the velocity field from the interplay of the totality of all of the system's velocity channels. We have termed the framework of our approach a "superclassical" one, because in it are combined classical levels at vastly different scales, i.e., at the subquantum and the macroscopic levels, respectively.

\section{Identity of the emergent force on a particle modeled by a bouncer system and the quantum force of the deBroglie-Bohm theory}

With the results of the foregoing Chapter, we can now return to and resolve the problem discussed in Sect. 1 of the apparent incompatibility between the Bohmian force upon a quantum particle and the force exerted on a bouncing droplet as formulated by Richardson et al. [20]. In fact, already a first look at the bouncer/walker model of our group provides a clear difference as compared to the hydrodynamical force studied by Richardson et al. Whereas the latter investigates the effects of essentially a single bounce on the fluid surface and the acceleration of the bouncer as a consequence of this interaction, our bouncer/walker model for quantum particles involves a much more complex dynamical scenario: we consider the effects of a huge number of bounces, i.e., typically of the order of $1 / \omega$, like approximately $10^{21}$ bounces per second of an electron, which constitute effectively a "heating up" of the bouncer's surrounding, i.e., the subquantum medium related to the zero-point energy field.

Note that as soon as a microdynamics is assumed, the development of heat fluxes is a logical necessity if the microdynamics is constrained by some macroscopic boundaries like that of a slit system, for example. As we have shown in some detail [12], the thermal field created by such a huge number of bounces in a slit system leads to an emergent average behavior of particle trajectories which is identified as anomalous, and more specifically as ballistic, diffusion. As such, the particle trajectories exiting from, say, a Gaussian slit behave exactly as if they were subject to a Bohmian quantum force. We were also able to show that this applies also to $n$-slit systems, such that one arrives at a subquantum modeling of the emergent interference effects at $n$ slits whose predicted average behavior is identical to that provided by the $\mathrm{dBB}$ theory.

It is then easily shown that the average force acting on a particle in our model is the same as the Bohmian quantum force. Due to the identity of our emerging velocity field with the guidance formula, and because they essentially differ only via the notations due to different forms of bookkeeping, their respective time derivatives must also be identical. Thus, from Eq. (7), one obtains the particle acceleration field (using a one-particle scenario for simplicity) in an $n$-slit system as 


$$
\begin{aligned}
a_{\mathrm{tot}}(t) & =\frac{\mathrm{d} \mathbf{v}_{\mathrm{tot}}}{\mathrm{d} t}=\frac{\mathrm{d}}{\mathrm{d} t}\left(\frac{\sum_{i=1}^{3 n} \mathbf{w}_{i} P\left(\mathbf{w}_{i}\right)}{\sum_{i=1}^{3 n} P\left(\mathbf{w}_{i}\right)}\right) \\
& =\frac{1}{\left(\sum_{i=1}^{3 n} P\left(\mathbf{w}_{i}\right)\right)^{2}}\left\{\sum_{i=1}^{3 n}\left[P\left(\mathbf{w}_{i}\right) \frac{\mathrm{d} \mathbf{w}_{i}}{\mathrm{~d} t}+\mathbf{w}_{i} \frac{\mathrm{d} P\left(\mathbf{w}_{i}\right)}{\mathrm{d} t}\right]\left(\sum_{i=1}^{3 n} P\left(\mathbf{w}_{i}\right)\right)-\left(\sum_{i=1}^{3 n} \mathbf{w}_{i} P\left(\mathbf{w}_{i}\right)\right)\left(\sum_{i=1}^{3 n} \frac{\mathrm{d} P\left(\mathbf{w}_{i}\right)}{\mathrm{d} t}\right)\right\} .
\end{aligned}
$$

Note in particular that (14) typically becomes infinite for regions $(\mathbf{x}, t)$ where $P_{\text {tot }}=\sum_{i=1}^{3 n} P\left(\mathbf{w}_{i}\right) \rightarrow 0$, in accordance with the Bohmian picture.

From (14), we see that even the acceleration of one particle in an $n$-slit system is a highly complex affair, as it nonlocally depends on all other accelerations and temporal changes in the probability densities across the whole experimental setup! In other words, this force is truly emergent, resulting from a huge amount of bouncermedium interactions, both locally and nonlocally. This is of course radically different from the scenario studied by Richardson et al. where the effect of only a single local bounce is compared with the quantum force. From our new perspective, it is then hardly a surprise that the comparison of the two respective forces provides distinctive differences. However, as we just showed, with the emergent scenario proposed in our model, complete agreement with the Bohmian quantum force is established.

\section{Choose your poison: how to introduce nonlocality in a hydrodynamic-like model for quantum systems?}

As already mentioned in the introduction of this paper, purely classical hydrodynamical models are manifestly local and thus inadequate tools to explain quantum mechanical nonlocality. Although nonlocal correlations may also be obtainable within hydrodynamical modeling [2], there is no way to also account for dynamical nonlocality [21] in this manner. So, as correctly observed by Richardson et al. [20], droplet-surface wave interaction scenarios are not enough to serve as a full-fledged analogy of the distinctly nonlocal dBB theory, for example.

The question thus arises how in our much more complex, but still "hydrodynamic-like" bouncer/walker model nonlocal, or nonlocal-like, effects can come about. To answer this, one needs to consider in more detail how the elements of our model are constructed, which finally provide an elegant formula, Eq. (7), identical with the guidance formula in a (for simplicity: one-particle) system with $n$ slits. (As shown above, the extension to $N$ particles is straightforward.) As we consider, without restriction of generality, the typical example of Gaussian slits, we introduce the Gaussians in the usual way, with $\sigma$ related to the slit width, for the probability density distributions (which in our model coincide with "heat" distributions due to the bouncers' stirring up of the vacuum) just behind the slit. The important feature of these Gaussians is that we do not implement any cutoff for the distributions, but maintain the long tails which actually then extend across the whole experimental setup, even if these are only very small and practically often negligible amplitudes in the regions far away from the slit proper. As the emerging probability density current is given by the denominator of Eq. (8), we see that in fact the product $R_{1} R_{2}$ may be negligibly small for regions where only a long tail of one Gaussian overlaps with another Gaussian, nevertheless the last term in (8) can be very large despite the smallness of $R_{1}$ or $R_{2}$. It is this latter part which is responsible for the genuinely quantum-like nature of the average momentum, i.e., for its nonlocal nature. This is similar in the Bohmian picture, but here given a more direct physical meaning in that this last term refers to a difference in diffusive currents as explicitly formulated in the last term of (8). Because of the mixing of diffusion currents from both channels, we call this decisive term in $\mathbf{J}_{\text {tot }}=\mathbf{P}_{\text {tot }} \mathbf{v}_{\text {tot }}$ the "entangling current" [16]. 
Thus, one sees that formally one obtains genuine quantum mechanical nonlocality in a hydrodynamic-like model with one particular "unusual" characteristic: the extremely feeble but long tails of (Gaussian or other) distribution functions for probability densities exiting from a slit extend nonlocally across the whole experimental setup. So, we have nonlocality by explicitly putting it into our model. After all, if the world is nonlocal, it would not make much sense to attempt its reconstruction with purely local means. Still, so far we have just stated a formal reason for how nonlocality may come about. Somewhere in any theory, so it seems, one has to "choose one's poison" that would provide nonlocality in the end. But what would be a truly "digestible" physical explanation? Here is where at present only some speculative clues can be given.

For one thing, strict nonlocality in the sense of absolute simultaneity of space-like separated events can never be proven in any realistic experiment, because infinite precision is not attainable. This means, however, that very short time lapses must be admitted in any operational scenario, with two basic options remaining: (1) either there is a time lapse due to the finitely fast "switching" of experimental arrangements in combination with instantaneous information transfer [but not signaling; see Walleczek and Grössing (forthcoming)], or (2) the information transfer itself is not instantaneous, but happens at very high speeds $v \ggg c$.

How, then, can the implementation of nonlocal or nonlocal-like processes with speeds $v \ggg c$ be argued for in the context of a hydrodynamic-like bouncer/walker model? We briefly mention two options here. Firstly, one can imagine that the "medium" we employ in our model is characterized by oscillations of the zero-point energy throughout space, i.e., between any two or more macroscopic boundaries as given by experimental setups. Between these boundaries standing wave configurations may emerge (similar to the Paris group's experiments, but now explicitly extending synchronously over nonlocal distances). Here it might be helpful to remind ourselves that we deal with solutions of the diffusion (heat conduction) equation. At least (but perhaps only) formally, any change of the boundary conditions is effective "instantaneously" across the whole setup. Alternatively, if the experimental setup is changed such that old boundary conditions are substituted by new ones, due to the all-space-pervading zero-point energy oscillations, one "immediately" (i.e., after a very short time of the order $t \sim \frac{1}{\omega}$ ) obtains a new standing wave configuration that now effectively implies an almost instantaneous change of probability density distributions, or relative phase changes, for example. The latter would then become "immediately" effective in that changed phase information is available across the whole domain of the extended probability density distribution. We have referred to this state of affairs as "systemic nonlocality" [14]. So, one may speculate that it is something like "eigenvalues" of the universe's network of zero-point fluctuations that may be responsible for quantum mechanical nonlocality-eigenvalues which (almost?) instantaneously change whenever the boundary conditions are changed.

A second option even more explicitly refers to the universe as a whole, or, more particularly, to spacetime itself. If spacetime is an emergent phenomenon as some recent work suggests [19], this would very likely have strong implications for the modeling and understanding of quantum phenomena. Just as in our model of an emergent quantum mechanics we consider quantum theory as a possible limiting case of a deeper level theory, present-day relativity and concepts of spacetime may be approximations of, and emergent from a superclassical, deeper level theory of gravity and/or spacetime. It is thus a potentially fruitful task to bring both attempts together in the near future.

Acknowledgments We thank Jan Walleczek for many enlightening discussions, and the Fetzer Franklin Fund for partial support of the current work.

\section{References}

1. Bohm, D.: Causality and Chance in Modern Physics. Routledge, London (1997)

2. Brady, R., Anderson, R.: Violation of bell's inequality in fluid mechanics (pre-print) (2013). arXiv:1305.6822 [physics.gen-ph]

3. Bush, J.W.: Pilot-wave hydrodynamics. Annu. Rev. Fluid. Mech. 47, 269-292 (2015). doi:10.1146/annurev-fluid-010814-014506

4. Couder, Y., Fort, E.: Single-particle diffraction and interference at a macroscopic scale. Phys. Rev. Lett. 97, 154101 (2006). doi:10. 1103/PhysRevLett.97.154101

5. Couder, Y., Fort, E.: Probabilities and trajectories in a classical wave-particle duality. J. Phys. Conf. Ser. 361, 012001 (2012). doi:10.1088/1742-6596/361/1/012001 
6. Fort, E., Eddi, A., Boudaoud, A., Moukhtar, J., Couder, Y.: Path-memory induced quantization of classical orbits. PNAS 107, 17515-17520 (2010). doi:10.1073/pnas.1007386107

7. Fussy, S., Mesa Pascasio, J., Schwabl, H., Grössing, G.: Born's rule as signature of a superclassical current algebra. Ann. Phys. 343, 200-214 (2014). doi:10.1016/j.aop.2014.02.002

8. Grössing, G.: The vacuum fluctuation theorem: exact Schrödinger equation via nonequilibrium thermodynamics. Phys. Lett. A 372, 4556-4563 (2008). doi:10.1016/j.physleta.2008.05.007

9. Grössing, G.: On the thermodynamic origin of the quantum potential. Physica A 388, 811-823 (2009). doi:10.1016/j.physa.2008. 11.033

10. Grössing, G. (ed.): Emergent Quantum Mechanics 2011. 361/1. IOP Publishing, Bristol (2012). http://iopscience.iop.org/ 1742-6596/361/1

11. Grössing, G., Elze, H.T., Mesa Pascasio, J., Walleczek, J. (eds.): Emergent Quantum Mechanics 2013. 504/1. IOP Publishing, Bristol (2014). http://iopscience.iop.org/1742-6596/504/1

12. Grössing, G., Fussy, S., Mesa Pascasio, J., Schwabl, H.: Emergence and collapse of quantum mechanical superposition: orthogonality of reversible dynamics and irreversible diffusion. Physica A 389, 4473-4484 (2010). doi:10.1016/j.physa.2010.07.017

13. Grössing, G., Fussy, S., Mesa Pascasio, J., Schwabl, H.: An explanation of interference effects in the double slit experiment: classical trajectories plus ballistic diffusion caused by zero-point fluctuations. Ann. Phys. 327, 421-437 (2012). doi:10.1016/j.aop. 2011.11.010

14. Grössing, G., Fussy, S., Mesa Pascasio, J., Schwabl, H.: Systemic nonlocality' from changing constraints on sub-quantum kinematics. J. Phys. Conf. Ser. 442, 012012 (2013). doi:10.1088/1742-6596/442/1/012012

15. Grössing, G., Fussy, S., Mesa Pascasio, J., Schwabl, H.: Relational causality and classical probability: grounding quantum phenomenology in a superclassical theory. J. Phys. Conf. Ser. 504, 012006 (2014). doi:10.1088/1742-6596/504/1/012006

16. Mesa Pascasio, J., Fussy, S., Schwabl, H., Grössing, G.: Modeling quantum mechanical double slit interference via anomalous diffusion: independently variable slit widths. Physica A 392, 2718-2727 (2013). doi:10.1016/j.physa.2013.02.006

17. Norsen, T.: The theory of (exclusively) local beables. Found. Phys. 40, 1858-1884 (2010). doi:10.1007/s10701-010-9495-2

18. Norsen, T., Marian, D., Oriols, X.: Can the wave function in configuration space be replaced by single-particle wave functions in physical space? Synthese (2014). doi:10.1007/s11229-014-0577-0

19. Padmanabhan, T.: General relativity from a thermodynamic perspective. Gen. Relativ. Gravit. 46 (2014). doi:10.1007/ s10714-014-1673-7

20. Richardson, C.D., Schlagheck, P., Martin, J., Vandewalle, N., Bastin, T.: On the analogy of quantum wave-particle duality with bouncing droplets (pre-print) (2014). arXiv:1410.1373 [physics.flu-dyn]

21. Tollaksen, J., Aharonov, Y., Casher, A., Kaufherr, T., Nussinov, S.: Quantum interference experiments, modular variables and weak measurements. New J. Phys. 12, 013023 (2010). doi:10.1088/1367-2630/12/1/013023 